\title{
A Framework for Sensitivity Analysis of Data Errors on Home Energy Management System
}

\author{
Dae-Hyun Choi ${ }^{a, *}$, Le Xie ${ }^{b}$ \\ ${ }^{a}$ School of Electrical and Electronics Engineering, Chung-ang University, Dongjak-gu, Seoul \\ 156-756, Republic of Korea \\ ${ }^{b}$ Department of Electrical and Computer Engineering, Texas A\&M University, College \\ Station TX, USA
}

\begin{abstract}
This paper investigates the impact of data errors on home energy management systems (HEMSs) that reduce energy cost and maintain comfort for residential consumers. In particular, we conduct a sensitivity analysis of HEMS subject to various types of input data such as the predicted energy consumption, the forecasted outdoor temperature, the consumers' comfort settings, static and dynamic operation constraints for home appliances, and the demand response (DR) signal. Using the perturbed Karush-Kuhn-Tucker (KKT) condition equations from the HEMS optimization formulation, we develop a linear sensitivity matrix to assess the impact of data on optimal solutions for: (1) electricity cost; (2) consumer's dissatisfaction cost; (3) the energy consumption for home appliances; and (4) the indoor temperature. The results of a simulation study using the developed sensitivity matrix provide HEMS operators with unique insight into factors that account for the relationships of HEMS operations to the change in the various data. Furthermore, these results can be used to provide insights for residential consumers and to evaluate the security risks of HEMS to cyber attacks through data manipulation.
\end{abstract}

Keywords: Home energy management system (HEMS), energy consumption scheduling, demand side management, sensitivity analysis.

\footnotetext{
* Corresponding author

Email address: dhchoi@cau.ac.kr (Dae-Hyun Choi)
}

Preprint submitted to Energy

July 30, 2016 


\section{Introduction}

There has been significant increase in smart energy appliances such as air conditioners, washers, and refrigerators. Furthermore, smart meters and distributed energy resources (DERs) (e.g., solar and energy storage systems) are 5 deployed in a smart grid based on an advanced metering infrastructure (AMI). It is therefore necessary to develop systems with which a utility is provided for consumers to manage their residential energy consumption for a reduction in household CO2 emission, energy savings, and efficiency of home appliances.

Home energy management systems (HEMSs) are the core technology for residential energy management in smart grids. The main functions of HEMS include: monitoring the real-time energy usage of consumers using smart meters and smart plugs, optimizing and scheduling the energy consumption of home appliances, and reducing consumers' electricity bills while satisfying consumers' comforts and preferences. It should be noted that the operation of

15 HEMS strongly relies on various types of sensor data associated with energy consumption - from smart meters, weather information (e.g., outdoor temperature), operational parameters for appliances such as power ratings, comfort settings by consumers such as preferred indoor temperature range, and demand response (DR) signals from a utility such as a demand reduction request (KW)

20 and duration (hours). Obviously, the change in these data caused by inherent and/or human attack could result in the serious malfunction of HEMS.

Maintaining the quality of data in a smart grid is becoming a crucial issue, which was well expressed by Gib Sorebo, chief cyber-security technologist at Science Applications International Corporation, "Even small changes in the data could affect the stability of the grid and even jeopardize human safety" [1]. This message is primarily relevant for operators of transmission and distribution systems whose primary role is to ensure reliable and secure grid functioning, although it is also relevant for HEMS operators of the last-mile distribution network. For example, the cyber security concern about HEMS has recently been addressed, where an adversary can stealthily manipulate data related to 
appliance energy consumption, a DR event, and priority/preference settings, consequently providing incorrect energy consumption scheduling for appliances to consumers or incorrect household consumption data to a utility [2].

The main objective of this paper is to study the sensitivity of HEMS to small changes in various input data caused by inherent and/or human disruptions (e.g., low accuracy sensors, adversary attacks, etc.). Fig. 1 illustrates the conceptual system model for HEMS, along with the data-dependent HEMS optimization process and the proposed sensitivity framework. Based on input data a, HEMS computes the optimal energy consumption of each home appliance $\mathbf{x}^{*}$ 40 and controls its operation according to $\mathrm{x}^{*}$. The novelty of this paper is that it proposes an analytical framework with which to evaluate the sensitivity of the optimal solution from HEMS in response to changes in five different types of data: (1) predicted energy demand; (2) operational constraints of appliances; (3) DR signals; (4) consumer's comfort setting; and (5) outdoor temperature.

45 Many recent papers have been presented on the subject of home energy management in smart grids. In [3, 4], the authors characterized the detailed load models of controllable major home appliances associated with demand response. They then developed an algorithm to schedule operations for maintaining the desired total power consumption using the consumers' preset priorities and preferences. A large amount of research has recently been conducted on optimization algorithms for HEMS: with a distributed energy resource (DER) [5, 6, 7, 8, 9, 10, 11, 12], based on particle swarm optimization [5], using mixed integer linear programming [6, 7, 8], using convex optimization [9, 10], using mixed integer nonlinear programming based on a relaxed convex optimiza-

55 tion with an $L_{1}$ regularization term [11], and considering a balance between the energy saving and a comfortable lifestyle [12]. Distributed HEMS algorithms for both the utility company and consumers have been proposed in [13, 14]. A literature survey on the recent HEMS optimization problem was summarized well in [15]. More recently, the impact of an optimization interval on HEMS ${ }_{60}$ was investigated [16] and a novel HEMS algorithm with voltage control was proposed, which led to the minimization of the load shifting operation [17]. It 
is noted that the aforementioned optimization-based HEMS algorithms involve various sets of data for the optimal energy consumption scheduling of appliances. Therefore, maintaining high-quality data improves the performance of

65 HEMS, and a data quality assessment tool is required for HEMS operators to ensure efficient and secure HEMS operation.

To the best of our knowledge, no study has been presented concerning the degree to which changes in data influence HEMS operations. This paper is therefore a first attempt that we know of to conduct an impact analysis of

70 HEMS subject to data errors. Many existing works have proposed a perturbation approach to data sensitivity analysis in the economic dispatch problem at the transmission level of an electric power system: with Lagrangian relaxationbased analysis with storage, network, and inter-temporal ramp constraints [18] and with the DC and AC optimal power flow models [19, 20] for a study of locational marginal price (LMP) sensitivity. Our proposed sensitivity framework is based on a perturbation approach using Karush-Kuhn-Tucker (KKT) conditions for general nonlinear optimization problems [21], which has been verified and illustrated in [20]. In this paper, we apply this perturbation approach at the transmission level to the HEMS model at the distribution level. We derive

so the desired sensitivity matrix, validate its feasibility, present meaningful observations through simulation studies, and suggest potential applications of the proposed approach. The main contributions of this paper are two-fold:

- We propose an analytical framework to assess the sensitivity of HEMS to changes in various types of data. We derive the linear sensitivity matrix based on the perturbed KKT equations from the HEMS optimization formulation. This matrix explains the relationship between the optimal solution and the input data in HEMS. The proposed framework is a closedform online analysis tool with a linear sensitivity matrix, offering HEMS operators a quick and accurate method to predict and analyze the performance of HEMS with varying data. From an implementation perspective, the advantage of the proposed approach is that the developed framework 
can be applicable to any HEMS optimization problem and readily integrated into the existing HEMS without the need to modify the HEMS.

- We perform rigorous simulation studies on the proposed sensitivity ap-

\subsection{Preliminary}

In this paper, we consider the scenario where the HEMS schedules the operation of two types of controllable loads: (1) reducible loads such as air conditioners, known as thermostatically controllable loads (TCLs), and shiftable mal day-ahead scheduling of these controllable appliances under Time-of-Use (TOU) pricing. We assume that HEMS is equipped with all input data prior to the optimal energy consumption scheduling. For example, the energy consumption data are collected, processed, and delivered by sensors (e.g., smart meter 
and smart plug) to HEMS and a prediction module 22] in HEMS uses these data to forecast appliance energy consumption and the consumer's consumption pattern. The main notations used throughout this paper are summarized in Table 1. Bold symbols represent vectors or matrices. Hat symbols represent predictions of true parameter values.

\subsection{HEMS Formulation}

In general, an algorithm for HEMS is formulated as an optimization problem with equality and inequality constraints as follows:

$$
\min _{\mathbf{x}} J(\mathbf{x}, \mathbf{a})
$$

s.t.

$$
\begin{aligned}
& \mathbf{f}(\mathbf{x}, \mathbf{a})=\mathbf{0} \\
& \mathrm{g}(\mathbf{x}, \mathbf{a}) \leq \mathbf{0}
\end{aligned}
$$

where $J(\mathbf{x}, \mathbf{a})$ is a scalar objective function, consisting of electricity cost $J_{1}(\mathbf{x}, \mathbf{a})$ and the consumer's dissatisfaction cost $J_{2}(\mathbf{x}, \mathbf{a}), \mathbf{f}(\mathbf{x}, \mathbf{a})$ are equality constraints for the balance equations of the predicted appliance energy consumption and operational dynamics of the appliances. $\mathbf{g}(\mathbf{x}, \mathbf{a})$ are inequality constraints for the physical capacity of the appliance operation and the consumer's comfort range.

In this paper, the HEMS algorithm is formulated as a quadratic programming (QP) optimization problem with linear equality and inequality constraints $(\mathbf{F x}=\mathbf{a}$ and $\mathbf{G x} \leq \mathbf{a})$. In the QP problem, the sensitivity of HEMS in response 135 to the change in data is rigorously investigated.

In the formulated optimization problem, the decision variable vector $\mathbf{x}$ and the data vector a contain the following elements:

$$
\begin{aligned}
& \mathbf{x}=\left(\mathbf{P}, \mathbf{A}^{\text {in }}\right), \\
& \mathbf{a}=\left(\boldsymbol{\omega}, A^{\text {comf }}, \widehat{\mathbf{D}}_{i}, \widehat{\mathbf{D}}_{t}, \alpha, \beta, \widehat{\mathbf{A}}^{\text {out }}, \Delta \mathrm{DR}, \mathbf{P}^{\text {cap }}, \mathbf{A}^{\text {in }, \text { cap }}\right),
\end{aligned}
$$


where

$$
\begin{aligned}
& \mathbf{P}_{\left(1 \times N_{P}\right)}=\left[P_{i, t}\right], \mathbf{A}_{\left(1 \times N_{A}\right)}^{\mathrm{in}}=\left[A_{t}^{\mathrm{in}}\right], \\
& \boldsymbol{\omega}=\left[\omega_{1} \omega_{2}\right], \widehat{\mathbf{D}}_{i\left(1 \times N_{\mathcal{I}}\right)}=\left[\widehat{D}_{i}\right], \widehat{\mathbf{D}}_{t\left(1 \times N_{\mathcal{T}}\right)}=\left[\widehat{D}_{t}\right], \widehat{\mathbf{A}}_{\left(1 \times N_{\mathcal{T}}\right)}^{\text {out }}=\left[\widehat{A}_{t}^{\text {out }}\right], \\
& \mathbf{P}_{\left(1 \times\left[2 N_{P}\right]\right)}^{\mathrm{cap}}=\left[\mathbf{P}^{\max T} \mathbf{P}^{\min T}\right], \mathbf{A}_{\left(1 \times\left[2 N_{A}\right]\right)}^{\mathrm{in}, \operatorname{cap}}=\left[\mathbf{A}^{\mathrm{in}, \max T} \mathbf{A}^{\mathrm{in}, \min T}\right] .
\end{aligned}
$$

The data vector a is categorized into five groups $((\mathrm{G} 1) \sim(\mathrm{G} 5))$ as shown in Table 2. (G1) and (G5) include estimates of energy consumption and outdoor temperature, based on smart meter (or smart plug) data and weather information. In (G2), the data are constant parameters determined by the home environment and the type of appliance. (G3) corresponds to an interaction with a utility. In (G4), the data are variable parameters that can be chosen by the consumer's comfort level and preference. The detailed objective function and equality/inequality constraints in the HEMS optimization formulation are expressed as follows:

\subsubsection{Objective function}

The objective function of the HEMS optimization problem is decomposed into two functions: electricity cost $J_{1}(\mathbf{x}, \mathbf{a})$ and the consumer's dissatisfaction $\operatorname{cost} J_{2}(\mathbf{x}, \mathbf{a})$ :

$$
J(\mathbf{x}, \mathbf{a})=\underbrace{\sum_{t \in \mathcal{T}} C_{t}\left(\sum_{i \in \mathcal{I}} P_{i, t}\right)}_{J_{1}(\mathbf{x}, \mathbf{a})}+\underbrace{\sum_{t \in \mathcal{T}}\left[\omega_{1}\left(A_{t}^{\mathrm{in}}-A^{\mathrm{comf}}\right)^{2}-\omega_{2}\right]}_{J_{2}(\mathbf{x}, \mathbf{a})} .
$$

The dissatisfaction cost represents the consumer's discomfort in terms of the difference between the indoor temperature in the house and the preferred temperature selected by the consumer [11]. In this paper, this cost corresponds to the operation of the air conditioner.

2.2.2. Equality constraint $(\mathbf{F} \mathbf{x}=\mathbf{a})$

For all $t$, the energy consumption balance equations, with the energy consumption prediction for the reducible and shiftable appliances, are expressed as, 
respectively,

$$
\begin{aligned}
\lambda^{(1)}: \sum_{t \in \mathcal{T}} P_{i, t} & =\widehat{D}_{i}-\Delta \mathrm{DR}, & & i \in \mathcal{I}_{r} \\
\lambda_{\left(N_{s} \times 1\right)}^{(2)}: \sum_{t \in \mathcal{T}} P_{i, t} & =\widehat{D}_{i}, & & i \in \mathcal{I}_{s} .
\end{aligned}
$$

It is noted that (7) incorporates an amount of the requested DR quantity ( $\Delta \mathrm{DR})$ because the total consumption of the reducible appliance must be curtailed with $\Delta \mathrm{DR}$ for $\mathrm{DR}$ duration. On the other hand, the total consumption of the shiftable appliance must stay the same irrespective of the DR signal. In this paper, we assume that the house has a single reducible appliance (i.e., the air conditioner).

The linear dynamics for the air conditioner's operation at $t$ is written in terms of the indoor/outdoor temperature, the air conditioner's power consumption and the parameters specifying the indoor thermal condition as in [11]

$$
\boldsymbol{\lambda}_{\left(N_{\mathcal{T} \times 1)}\right.}^{(3)}: A_{t}^{\text {in }}=A_{t-1}^{\text {in }}+\alpha\left(\widehat{A}_{t}^{\text {out }}-A_{t-1}^{\text {in }}\right)+\beta P_{i, t}, \quad i \in \mathcal{I}_{r} .
$$

For all controllable appliances, the DR balance equation associated with the DR period $\left(t \in \mathcal{T}_{\mathrm{DR}}\right)$ is expressed as

$$
\lambda^{(4)}: \sum_{i \in \mathcal{I}} \sum_{t \in \mathcal{T}_{\mathrm{DR}}} P_{i, t}=\sum_{t \in \mathcal{T}_{\mathrm{DR}}} \widehat{D}_{t}-\Delta \mathrm{DR} .
$$

\subsubsection{Inequality constraint $(\mathbf{G x} \leq \mathbf{a})$}

The constraints for physical appliance capacity and preferred temperature range are expressed as

$$
\begin{array}{lr}
\boldsymbol{\mu}_{\left(\left[2 N_{P}\right] \times 1\right)}^{(1)}: P_{i}^{\min } \gamma_{i, t} \leq P_{i, t} \leq P_{i}^{\max } \gamma_{i, t}, \quad i \in \mathcal{I}, t \in \mathcal{T} \\
\boldsymbol{\mu}_{\left(\left[2 N_{A}\right] \times 1\right)}^{(2)}: A^{\text {in,min }} \leq A_{t}^{\text {in }} \leq A^{\text {in,max }}, & t \in \mathcal{T} .
\end{array}
$$

\section{Proposed Sensitivity Framework for HEMS}

The primary goal of this paper is to provide an analytical framework to assess the sensitivity of electricity cost, the dissatisfaction cost, the optimal energy consumption of home appliances and indoor temperature with respect 
to the changes in the five types of HEMS data as classified in the previous section:

$$
\frac{\partial J_{1}}{\partial \mathbf{a}}, \frac{\partial J_{2}}{\partial \mathbf{a}}, \frac{\partial \mathbf{P}}{\partial \mathbf{a}}, \frac{\partial \mathbf{A}^{\text {in }}}{\partial \mathbf{a}} .
$$

To this end, using the Karush-Kuhn-Tucker (KKT) conditions we apply a perturbation approach 21] to the HEMS optimization problem formulated in Section 2, The Lagrangian function of the HEMS optimization problem is formulated as

$$
\begin{aligned}
\mathcal{L} & =J_{1}(\mathbf{x}, \mathbf{a})+J_{2}(\mathbf{x}, \mathbf{a})-\lambda^{(1)}\left(\sum_{t=1}^{N_{\mathcal{T}}} P_{i, t}-\widehat{D}_{i}+\Delta \mathrm{DR}\right)-\sum_{i=1}^{N_{\mathcal{I}_{s}}} \boldsymbol{\lambda}_{i}^{(2)}\left(\sum_{t=1}^{N_{\mathcal{T}}} P_{i, t}-\widehat{D}_{i}\right) \\
& -\sum_{t=1}^{N_{\mathcal{T}}} \boldsymbol{\lambda}_{t}^{(3)}\left(A_{t}^{\mathrm{in}}-A_{t-1}^{\mathrm{in}}-\alpha\left(\widehat{A}_{t}^{\text {out }}-A_{t-1}^{\text {in }}\right)-\beta P_{i, t}\right) \\
& -\lambda^{(4)}\left(\sum_{i=1}^{N_{\mathcal{I}}} \sum_{t=1}^{N_{\mathcal{T}_{\mathrm{DR}}}} P_{i, t}-\sum_{t=1}^{N_{\mathcal{T}_{\mathrm{DR}}}} \widehat{D}_{t}+\Delta \mathrm{DR}\right) \\
& +\sum_{j=1}^{2 N_{P}} \mu_{j}^{(1)} \sum_{i=1}^{N_{P}}\left(Q_{j i} P_{i}-E_{j}\right)+\sum_{l=1}^{2 N_{A}} \mu_{l}^{(2)} \sum_{t=1}^{N_{A}}\left(S_{l t} A_{t}^{\text {in }}-F_{l}\right),
\end{aligned}
$$

where $Q_{j i}, S_{l t}, E_{j}$ and $F_{l}$ are the elements of the following matrices

$$
\begin{aligned}
& \mathbf{Q}_{\left(2 N_{P} \times N_{P}\right)}=\left[Q_{j i}\right]=\left[\begin{array}{c}
\operatorname{Diag}^{\left(N_{\mathcal{I}}\right)}\left(\mathbf{I}_{N_{\mathcal{T}}}\right) \\
\operatorname{Diag}^{\left(N_{\mathcal{I}}\right)}\left(-\mathbf{I}_{N_{\mathcal{T}}}\right)
\end{array}\right], \\
& \mathbf{S}_{\left(2 N_{A} \times N_{A}\right)}=\left[S_{l t}\right]=\left[\begin{array}{c}
\mathbf{I}_{N_{A}} \\
-\mathbf{I}_{N_{A}}
\end{array}\right], \\
& \mathbf{E}_{\left(2 N_{P} \times 1\right)}=\left[E_{j}\right]=\left[\begin{array}{c}
\hat{\mathbf{P}}^{\text {max }} \boldsymbol{\gamma} \\
-\hat{\mathbf{P}}^{\text {min }} \boldsymbol{\gamma}
\end{array}\right], \\
& \mathbf{F}_{\left(2 N_{A} \times 1\right)}=\left[F_{l}\right]=\left[\begin{array}{r}
\mathbf{A}^{\text {in,max }} \\
-\mathbf{A}^{\text {in,min }}
\end{array}\right] .
\end{aligned}
$$

Here, $\operatorname{Diag}^{(n)}(\mathbf{M})$ represents a block diagonal matrix consisting of $n$ blocks of the matrix M. Let us denote the total number of the binding inequality constraints for the appliance capacity and the preferred temperature range as $N_{B_{c}}$ and $N_{B_{a}}$, respectively. Then, the KKT first-order conditions with the optimal solution 
$\left(\mathbf{x}^{*}, \boldsymbol{\lambda}^{*}, \boldsymbol{\mu}^{*}\right)$ are expressed as

$$
\begin{aligned}
& \text { (C1) } \frac{\partial J_{1}\left(\mathbf{x}^{*}, \mathbf{a}\right)}{\partial P_{i, t}}-\lambda^{*(1)} \chi_{\left\{i \in \mathcal{I}_{r}\right\}}-\lambda^{*(2)} \chi_{\left\{i \in \mathcal{I}_{s}\right\}} \\
& +\lambda_{t}^{*(3)} \beta \chi_{\left\{i \in \mathcal{I}_{r}\right\}}-\lambda^{*(4)} \chi_{\left\{i \in \mathcal{I}_{s}\right\}}+\sum_{j=1}^{N_{B_{c}}} \mu_{j}^{*(1)} Q_{j i}=0, \quad \forall i, t \\
& \text { (C2) } \left.\frac{\partial J_{2}\left(\mathbf{x}^{*}, \mathbf{a}\right)}{\partial A_{t}^{\text {in }}}-\left[\lambda_{t}^{*(3)}-(1-\alpha) \lambda_{t+1}^{*(3)}\right)\right]+\sum_{l=1}^{N_{B a}} \mu_{l}^{*(2)} S_{l t}=0, \quad \forall t \\
& \text { (C3) } \sum_{t=1}^{N_{\mathcal{T}}} P_{i, t}^{*}=\widehat{D}_{i}-\Delta \mathrm{DR} \chi_{\left\{i \in \mathcal{I}_{r}\right\}}, \quad \forall i \\
& \text { (C4) } A_{t}^{* \text { in }}-A_{t-1}^{* \text { in }}-\alpha\left(\widehat{A}_{t}^{\text {out }}-A_{t-1}^{* \text { in }}\right)=\beta P_{i, t}^{*}, \quad i \in \mathcal{I}_{r}, \quad \forall t \\
& \text { (C5) } \sum_{i=1}^{N_{\mathcal{I}}} \sum_{t=1}^{N_{\mathcal{T}_{\mathrm{DR}}}} P_{i, t}^{*}=\sum_{t=1}^{N_{\mathcal{T}_{\mathrm{DR}}}} \widehat{D}_{t}-\Delta \mathrm{DR}, \\
& \text { (C6) } \sum_{i=1}^{N_{P}} Q_{j i} P_{i}^{*}=E_{j}, \quad \forall j=1, \ldots, N_{B_{c}} \\
& \text { (C7) } \sum_{t=1}^{N_{A}} S_{l t} A_{t}^{* \text { in }}=F_{l} \quad \forall l=1, \ldots, N_{B_{a}} .
\end{aligned}
$$

Here, $\chi_{\{i \in \mathcal{S}\}}$ is the characteristic function based on the set $\mathcal{S}$. In other words, $\chi_{\{i \in \mathcal{S}\}}=1$ when $i \in \mathcal{S}$; otherwise $\chi_{\{i \in \mathcal{S}\}}=0$. Therefore, the equations $(\mathrm{C} 1)$ and (C3) include the two KKT conditions, each of which corresponds to the reducible and shiftable appliances, respectively.

Next, all KKT equations $(\mathrm{C} 1) \sim(\mathrm{C} 7)$ together with the objective functions $J_{1}$ and $J_{2}$ are perturbed with respect to the decision variables $\left(P_{i, t}, A_{t}^{\text {in }}\right)$, Lagrange multipliers $\left(\lambda^{(1)}, \boldsymbol{\lambda}^{(2)}, \boldsymbol{\lambda}^{(3)}, \lambda^{(4)}, \mu_{j}^{(1)}, \mu_{l}^{(2)}\right)$ and data $\left(\boldsymbol{\omega}, A^{\text {comf }}, \widehat{D}_{i}, \widehat{D}_{t}, \alpha, \beta, \widehat{A}^{\text {out }}\right.$, $\left.\Delta \mathrm{DR}, E_{j}, F_{l}\right)$ while the KKT conditions still hold. For example, the perturbed equation of $(\mathrm{C} 7)$ is $\sum_{t=1}^{N_{A}} S_{l t} d A_{t}^{* \text { in }}=d F_{l}$. All perturbed equations are finally written in a linear matrix equation form (18). Taking the inverse of $\boldsymbol{\Lambda}$ on both sides of (18), we obtain the following sensitivity matrix

$$
\mathrm{S}_{\mathrm{M}}=\Lambda^{-1} \Omega
$$

, which provides the desired sensitivity information. The linear sensitivity matrix $\mathbf{S}_{\mathbf{M}}$ is the main result in this paper that can be used by HEMS operators 
to quantify the impact of all data sets on HEMS in an on-line manner. The in the Appendix $\mathrm{A}$

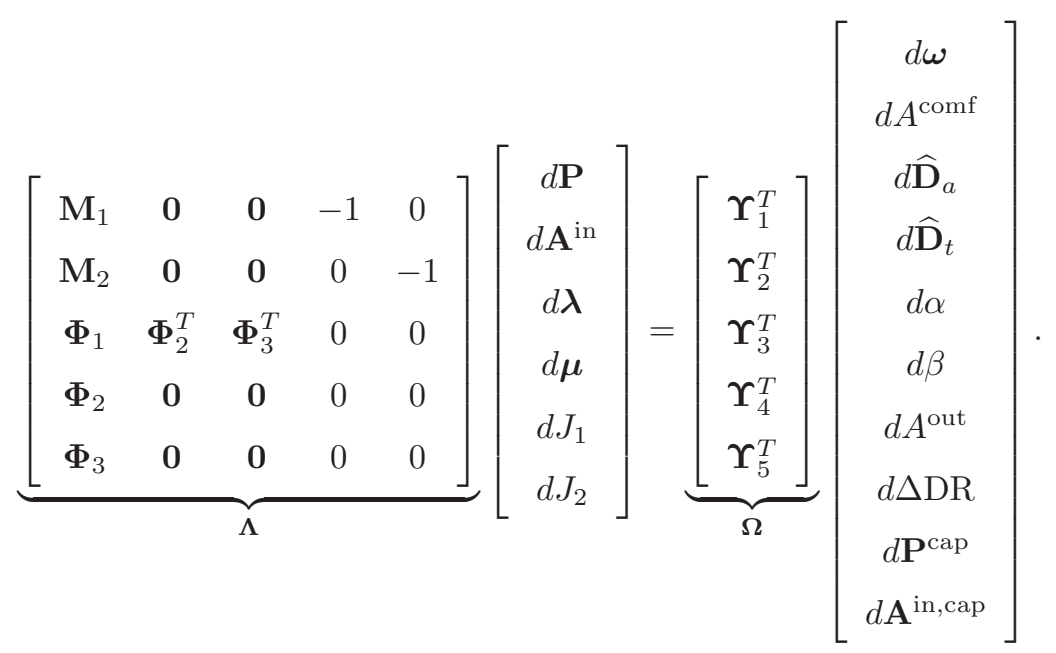

\section{Numerical Results}

In this section, we illustrate and verify the proposed matrix $\mathbf{S}_{\mathbf{M}}$ to evaluate the sensitivities of HEMS to changes in data. A simulation environment for the sensitivity analysis is described in Subsection 4.1. The sensitivity results are presented in two subsections. In Subsection 4.2, we compute the sensitivities of $P_{i, t}$ and $A_{t}^{\text {in }}$ to the three different data groups with the identical units $-\left(\omega_{1}, \alpha\right)$, $\left(\widehat{D}_{i}, \Delta \mathrm{DR}, P_{i, t}^{\max }\right)$ and $\left(\widehat{A}_{t}^{\text {out }}, A^{\mathrm{max}}, A^{\text {comf }}\right)$ - as follows:

$$
\begin{gathered}
\left\{\frac{\partial P_{i, t}}{\partial \omega_{1}}, \frac{\partial P_{i, t}}{\partial \alpha}\right\}(\mathrm{KW}), \quad\left\{\frac{\partial A_{t}^{\text {in }}}{\partial \omega_{1}}, \frac{\partial A_{t}^{\text {in }}}{\partial \alpha}\right\}\left({ }^{\circ} \mathrm{F}\right) \\
\left\{\frac{\partial P_{i, t}}{\partial \widehat{D}_{i}}, \frac{\partial P_{i, t}}{\partial \Delta \mathrm{DR}}, \frac{\partial P_{i, t}}{\partial P_{i, t}^{\max }}\right\},\left\{\frac{\partial A_{t}^{\text {in }}}{\partial \widehat{D}_{i}}, \frac{\partial A_{t}^{\text {in }}}{\partial \Delta \mathrm{DR}}, \frac{\partial A_{t}^{\text {in }}}{\partial P_{i, t}^{\max }}\right\}\left({ }^{\circ} \mathrm{F} / \mathrm{KW}\right) \\
\left\{\frac{\partial P_{i, t}}{\partial \widehat{A}_{t}^{\text {out }}}, \frac{\partial P_{i, t}}{\partial A^{\text {max }}}, \frac{\partial P_{i, t}}{\partial A_{t}^{\text {comf }}}\right\}\left(\mathrm{KW} /{ }^{\circ} \mathrm{F}\right),\left\{\frac{\partial A_{t}^{\text {in }}}{\partial \widehat{A}_{t}^{\text {out }}}, \frac{\partial A_{t}^{\text {in }}}{\partial A^{\text {max }}}, \frac{\partial A_{t}^{\text {in }}}{\partial A_{t}^{\text {comf }}}\right\} .
\end{gathered}
$$

Similarly, the following sensitivities for $J_{1}$ and $J_{2}$ are presented in Subsection 4.3

$$
\left\{\frac{\partial J_{1}}{\partial \widehat{D}_{i}}, \frac{\partial J_{1}}{\partial \Delta \mathrm{DR}}, \frac{\partial J_{1}}{\partial P_{i, t}^{\max }}\right\}(\$ / \mathrm{KWh}),
$$




$$
\begin{aligned}
& \left\{\frac{\partial J_{2}}{\partial \widehat{D}_{i}}, \frac{\partial J_{2}}{\partial \Delta \mathrm{DR}}, \frac{\partial J_{2}}{\partial P_{i, t}^{\max }}\right\}\left(\left({ }^{\circ} \mathrm{F}\right)^{2} / \mathrm{KWh}\right), \\
& \left\{\frac{\partial J_{1}}{\partial \widehat{A}_{t}^{\text {out }}}\right\}\left(\$ /{ }^{\circ} \mathrm{Fh}\right), \quad\left\{\frac{\partial J_{2}}{\partial \widehat{A}_{t}^{\text {out }}}\right\}\left({ }^{\circ} \mathrm{F}\right) .
\end{aligned}
$$

\subsection{Simulation setup}

We consider the situation where a household has five major home appliances (air conditioner, cloth dryer, washing machine, dish washer and oven, $i=1,2,3,4,5)$ that can be controlled by HEMS under the TOU tariff. For analytical purposes in the proposed sensitivity approach, the sensitivity matrix for a single-house scenario is derived, which could be extended for a multiplehouse scenario in the future work. The total scheduling period is one day with 24 time slots $\left(N_{\mathcal{T}}=24\right)$ so that each time slot is 1 hour. It is assumed that the household has joined a DR program and accepted a DR reduction request $(\Delta \mathrm{DR}=200 \mathrm{KW})$ within the period (14 P.M. $\sim 16$ P.M. $)$. The air conditioner is categorized as a reducible appliance $\left(\mathcal{I}_{r}\right)$; the other appliances are classified as shiftable appliances $\left(\mathcal{I}_{s}\right)$. The maximum power consumption $(\mathrm{KW})$ of each appliance is: $P_{1}^{\max }=1.25, P_{2}^{\max }=0.7, P_{3}^{\max }=0.65, P_{4}^{\max }=0.4$ and $P_{5}^{\max }=1$. The range of the consumer's comfortable temperature, the most comfortable temperature, and the initial indoor temperature are $A^{\text {in,max }}=79^{\circ} \mathrm{F}$, $A^{\text {in,min }}=70^{\circ} \mathrm{F}, A^{\text {comf }}=74^{\circ} \mathrm{F}$, and $A_{0}^{\text {in }}=76^{\circ} \mathrm{F}$ 14], respectively. The predicted outdoor temperature $\widehat{A}_{t}^{\text {out }}$ is obtained from [11]. The thermal and dissatisfaction cost parameters for the air conditioner are $\alpha=0.5, \beta=-0.008$ and $\omega_{1}=0.1, \omega_{2}=0.1$, respectively [14]. The consumer's consumption pattern is assumed to be as follows: $\gamma_{2, t}=1, t \in\left[5\right.$ A.M, 22 P.M.], $\gamma_{3, t}=1$, $t \in\left[6\right.$ A.M, 22 P.M.], $\gamma_{4, t}=1, t \in$ [6 A.M, 11 A.M.], $t \in$ [17 P.M, 24 A.M.], $\gamma_{5, t}=1, t \in[6$ A.M, 13 P.M. $], t \in[17$ P.M, 21 P.M. $]$. TOU rates are selected from Table I in [5]: $0.36 \$ /$ KWh for peak (2-8 P.M.), $0.14 \$ /$ KWh for shoulder (7 A.M - 2 P.M, 8 - 10 P.M.) and $0.14 \$ /$ KWh for off-peak (10 P.M - 7 A.M). 


\subsection{Sensitivity of the optimal appliance energy consumption and indoor tem- perature to data changes}

We first investigate the two types of sensitivities, $\partial P_{i, t} / \partial \omega_{1}$ and $\partial P_{i, t} / \partial \alpha$, as shown in Figs. 2 We observe from Figs. 2 that among the appliances the energy scheduling for the air conditioner is most affected by the varying $\omega_{1}$ and $\alpha$. This is expected because these parameters are used to determine the dissatisfaction cost and the dynamics only for the air conditioner. Comparing Fig. 2(a) and Fig. 2(b), Fig. 2(a) shows a high impact of $\omega_{1}$ on only the air conditioner at a few scheduling periods, whereas Fig. 2(b) shows a low impact of $\alpha$ on all appliances at a great number of scheduling periods. That is, the indoor thermal condition has lower but broader impacts on all appliances' energy scheduling than the consumer's discomfort level. These sensitivity results imply that without affecting the other appliances' energy scheduling, HEMS operators can adjust the energy scheduling of the air conditioner by tuning the parameter for the consumer's discomfort.

Figs. 3 show the sensitivities, $\partial P_{i, t} / \partial \widehat{D}_{4}, \partial P_{i, t} / \partial \Delta \mathrm{DR}$ and $\partial P_{i, t} / \partial P_{1,14}^{\max }$. With the perturbed (predicted) daily consumption $\widehat{D}_{4}$ for the dish washer, the sensitivities of the dish washer consumption $P_{4, t}$ at all scheduling periods are much greater than those of the other appliances and have positive values in Fig. 3(a). In our simulation, this observation holds true for different appliances, in which the predicted consumption data have a greater impact on the corresponding appliance's energy scheduling. In addition, using the sensitivities in the matrix $\mathbf{S}_{\mathbf{M}}$, HEMS operators can quickly identify the most vulnerable ap215 pliance among the five appliances to the change in the predicted consumption data. Since the prediction of the energy consumption of an individual appliance is conducted based on consumption data that are collected by the corresponding smart plug, this identification provides practical guidelines to select the order of upgrade or type of protection of smart plug from data attacks. Fig. 3(b) shows the sensitivity of $P_{i, t}$ to $\Delta \mathrm{DR}$. In contrast to the results in Fig. 33(a), we observe that the order of the sensitivity magnitude for all appliances varies according to different scheduling intervals. This is because the DR balance constraint in- 
volves the energy scheduling of all appliances. In Fig. 3(b), it is noted that the sensitivities for shiftable appliances in the DR period (14 P.M. 16 P.M.) are larger than in the non-DR period. This observation explains the TOU effect, where more $\mathrm{DR}$ reduction $(\Delta \mathrm{DR}>0)$ enables the shiftable appliances to operate in periods with lower prices $\left(\partial P_{i, t} / \partial \Delta \mathrm{DR}>0\right)$. We also observe that the sensitivities associated with the air conditioner are all 0 during the DR period since it already consumes the maximum power so that the given DR signal has no effect on the air conditioner's energy scheduling. The sensitivity results from Fig. 3(b) help HEMS operators to find appliances that are sensitive to DR signals, as well as predict the change of appliance energy scheduling with varying DR signals. In addition, since DR signals can be distorted by an adversary through communication networks between a utility and consumer [2], these sensitivity results could be valuable input data to assess security risks for residential DR programs. Fig. 3( 3 (c) shows that the change in $P_{1,14}^{\max }$ at $t=14$ results in the highest sensitivity of $P_{1, t}$. This is because the scheduled consumption of the air conditioner is binding at its maximum power rating during one of the DR periods, and the increase of the maximum power rating leads to the decrease of the energy consumption of the air conditioner with a negative sensitivity. The results in Fig. 3(b) and Fig. [3(c) are insightful, since they inform the HEMS operators that, given the HEMS operating condition, maintaining the quality of $P_{1,14}^{\max }$ at the $\mathrm{DR}$ period is more important than maintaining that of $\Delta \mathrm{DR}$ for a reliable energy consumption scheduling.

245 Fig. 4 shows the sensitivities of the air conditioner's consumption subject to data for the forecasted outdoor temperature $\widehat{A}_{11}^{\text {out }}$ at $t=11$ and the consumerselected comfortable temperature, $A_{11}^{\mathrm{in}, \max }$ and $A^{\text {comf }}$. We observe that the maximum comfortable temperature limit has a more significant impact on the sensitivity than the outdoor temperature. In this simulation, $A^{\text {comf }}$ was found to have no impact on the sensitivity. For the desired HEMS operations, system operators need to pay attention to the data accuracy associated with the consumer's preference rather than that associated with the weather information.

Next, we quantify the sensitivity of $A_{t}^{\text {in }}$ to data as shown in Figs. 囵 In 
Fig. [5)(a), the change of $\omega_{1}$ yields opposite sensitivities of $A_{t}^{\text {in }}$ to those of $P_{1, t}$ as shown in Fig. 2(a). This is because a trade-off relationship exists between $P_{1, t}$ and $A_{t}^{\text {in }}$ with respect to $\omega_{1}$. In addition, all sensitivities $\partial A_{t}^{\text {in }} / \partial \alpha$ were observed to have identical signs. This is to be expected because $\alpha$ is the coefficient for $\widehat{A}_{t}^{\text {out }}-A_{t-1}^{\text {in }}$ in the KKT equation $(\mathrm{C} 4)$, and in our simulation $\widehat{A}_{t}^{\text {out }}$ is greater than or equal to $A_{t-1}^{\text {in }}$ at each scheduling period $t$. In addition, some scheduling periods have $\partial A_{t}^{\text {in }} / \partial \alpha=0$. These periods correspond to $A_{t}^{\text {in }}$, which is binding at $A_{t}^{\text {in,max }}$ or $A_{t}^{\text {in,min }}$. The sensitivities of the indoor temperature to the predicted consumption of the air conditioner, the requested DR, and the maximum capacity of the air conditioner are shwon in Fig. 5 (b). From comparison of the plot for the air conditioner in Figs. 3(b),(c) and Fig. 5(b), we observe that the 265 sensitivities of $P_{1, t}$ and $A_{t}^{\text {in }}$ to both $\Delta \mathrm{DR}$ and $P_{1,14}^{\max }$ have opposite signs due to a trade off relationship between these variables. For example, the increase of $P_{1,14}^{\max }$ (or the decrease of $\Delta \mathrm{DR}$ ) leads to the increase of $P_{1, t}$ and the decrease of $A_{t}^{\text {in }}$. Lastly, the sensitivities of the indoor temperature with respect to the predicted outdoor temperature, the maximum comfortable temperature and the most comfortable temperature are shown in Fig. [5(c). From this figure, we can obtain the same observation as that in Fig. 4. That is, $A^{\text {in,max }}$ has the most significant impact on the calculation for $A_{t}^{\text {in }}$ among the three types of data; however, it yields sensitivities with the opposite sign to those for $P_{1, t}$.

In summary, similar to the sensitivity analysis of the appliance's consumption $P_{i, t}$, the sensitivity results for the indoor temperature $A_{t}^{\text {in }}$ to all data help HEMS operators to list the data in decreasing (or increasing) order of sensitivity and identify the most (or least) influential data to the consumer's comfort level. Using these sensitivity results, they could provide an energy consulting service for consumers in view of maintaining consumer's comfort. For example, using the results in Fig. 5(c), HEMS operators recommend that the consumer increases $A^{\text {in,max }}$ when needing to rapidly cool the house at 11 A.M. 


\subsection{Sensitivity of the optimal electricity bill and dissatisfaction cost to data changes}

Figs. [6] show the sensitivities of the optimal electricity bill and dissatisfaction cost to the predicted appliance consumption, the requested DR, the maximum capacity limit of appliances and the predicted outdoor temperature. We observe from Fig. 6(a) that only the sensitivity for $\Delta \mathrm{DR}$ is negative and the other sensitivities are positive. This is consistent with our expectation that a positive change in $\triangle \mathrm{DR}$ results in the decrease of the consumption of an appliance or a shift to operating periods with lower prices and a consequent reduction in the electricity bill. On the other hand, positive changes in $\widehat{D}_{i}$ and $P_{1,14}^{\max }$ lead to increases in the electricity bill with increasing appliance consumption. Comparing Fig. 6(a) with Fig. 6(b), the sensitivities subject to the same data have opposite signs due to a trade-off between the electricity bill and the dissatisfaction cost. We can conclude from these figures that $\Delta \mathrm{DR}$ and $P_{1,14}^{\max }$ are the most influential factors for electricity bill savings and for enhancing consumer's comfort, respectively. Also, the results from these figures can be used to provide an energy consulting service for the consumer to maximize electricity bill savings or the comfort level. Figs. 6(c),(d) show the sensitivities of $J_{1}$ and $J_{2}$ to $A_{t}^{\text {out }}$. In Fig. 6(c), we observe that the sensitivities of $J_{1}$ are somewhat larger in the scheduling periods, corresponding to high outdoor temperature. This implies that an increasing outdoor temperature requires increased operation of the air conditioner, consequently leading to an increased electricity bill. On the other hand, the sensitivities of $J_{2}$ with a positive sign appear to be almost evenly affected by $A_{t}^{\text {out }}$ as shown in Fig. 6(d). The results from this figure are consistent with our expectation since the increase of the outdoor temperature results in the increase of the consumer's discomfort. In particular, we verify from Fig. 6) (d) that the changes in temperature data at 10 A.M. and 7 A.M. have the most and least significant impacts on the consumer's discomfort, respectively.

Finally, the potential applications of the proposed sensitivity based approach can be summarized as follows: 
- Data error analysis tool: The derived closed-form sensitivity matrix provides HEMS operators with an analysis tool to quickly assess the impact of data errors on HEMS. This matrix can be readily integrated into the existing optimization-based HEMS and provide accurate sensitivity results of the optimal HEMS solution in an online manner. The sensitivity results can be potentially delivered to transmission and distribution system operators to ensure a reliable and secure grid operation.

- Energy consulting service: Using the results from the derived sensitivity matrix, HEMS operators can offer consumers a detailed energy consulting service to obtain the maximum electricity bill savings and consumer's comfort. This can be obtained through the identification of data that has the most influence on HEMS and by providing customized recommendations for adjusting such data.

- Cybersecurity assessment tool: The proposed sensitivity matrix can be chosen as a cybersecurity tool to assess the risks of cyber attack through the manipulation of HEMS data. The sensitivity results can also be used as valuable information to prioritize the upgrade of sensors that monitor energy usage, such as smart plugs and smart meters.

\section{Conclusions}

This paper proposes a novel analytical framework to quantify the sensitivity of HEMS to changes in input data for HEMS operation. In general, the proposed sensitivity framework can be applicable to any HEMS that performs home energy scheduling using an optimization algorithm. We develop a linear sensitivity matrix that describes the relationships in HEMS operation between optimal solutions (for the electricity bill, the consumer's dissatisfaction cost, appliance energy consumption, and indoor temperature) and data (energy consumption and operation for appliances, DR signals, comfort settings, weather information). The developed sensitivity matrix provides HEMS operators with 
340 an analytical tool to rigorously examine and compare the impact of data on HEMS. Practical guidelines are provided that could augment an energy consulting service for consumers or serve as a vulnerability assessment tool to secure HEMS against potential cyber data attacks. This work is the first analytical approach with a closed-form solution for rapidly assessing the impact of different types of data on HEMS operation.

In future work, a comprehensive sensitivity framework will be developed to study the impact of a broader set of data on an advanced HEMS that involves the operation of distributed energy resources (DERs). In this framework, based on the detailed modeling of DERs and home appliances, the data will represent the state of charge (SOC) and the charging/discharging efficiency for energy storage systems (ESSs), solar power, characteristics of controllable appliances, etc. Also, the developed framework would need to be simulated and verified in larger test systems with multiple houses under various and realistic circumstances, such as under different consumer profiles and pricing schemes such as critical peak pricing $(\mathrm{CPP})$ and real time pricing (RTP). Another interesting future direction is the design of a robust HEMS algorithm based on the data sensitivity matrix in order to detect and mitigate data attacks on HEMS.

\section{Acknowledgments}

This work was supported by the National Research Foundation of Korea 360 (NRF) grant funded by the Korea government (MSIP:Ministry of Science, ICT \& Future Planning) (No. 2015R1C1A1A01051890).

\section{A. PERTURBATION SUBMATRICES}

The submatrices in (18) are expressed in the following two subsections. 


\section{A.1. The Submatrices in the Left-hand Side of (18)}

The following two matrices correspond to the perturbed objective functions with respect to the decision variables:

$$
\begin{aligned}
& \mathbf{M}_{1\left(1 \times\left[N_{P}+N_{A}\right]\right)}=\left[\begin{array}{ll}
\left.\frac{\partial J_{1}\left(\mathbf{x}^{*}, \mathbf{a}\right)}{\partial P_{i, t}}\right]_{\left(1 \times N_{P}\right)} & \mathbf{0}_{N_{A}}^{T}
\end{array}\right], \\
& \mathbf{M}_{2\left(1 \times\left[N_{P}+N_{A}\right]\right)}=\left[\begin{array}{ll}
\mathbf{0}_{N_{P}}^{T} & {\left[2 \omega_{1}\left(A_{t}^{* \mathrm{in}}-A^{\mathrm{comf}}\right)\right]_{\left(1 \times N_{A}\right)}}
\end{array}\right] .
\end{aligned}
$$

The other three matrices are associated with the perturbed KKT condition equations with respect to the decision variables and Lagrange multipliers:

$$
\begin{aligned}
& \boldsymbol{\Phi}_{1\left(\left[N_{P}+N_{A}\right] \times\left[N_{P}+N_{A}\right]\right)}= \\
& {\left[\begin{array}{cc}
\operatorname{Diag}\left(\frac{\partial}{\partial P_{i, t}}\left(\frac{\partial J_{1}\left(\mathbf{x}^{*}, \mathbf{a}\right)}{\partial P_{i, t}}\right) d P_{i, t}\right) & \mathbf{0}_{\left(N_{P} \times N_{A}\right)} \\
\mathbf{0}_{\left(N_{A} \times N_{P}\right)} & \operatorname{diag}\left(2 \omega_{1}\right)_{\left(N_{A} \times N_{A}\right)}
\end{array}\right],} \\
& \boldsymbol{\Phi}_{2\left(\left[N_{P}+N_{A}\right] \times N_{e q}\right)}^{T}=\left[\begin{array}{cccc}
\boldsymbol{\Phi}_{2,1}^{T} & \boldsymbol{\Phi}_{2,2}^{T} & \boldsymbol{\Phi}_{2,3}^{T} & \boldsymbol{\Phi}_{2,4}^{T}
\end{array}\right]
\end{aligned}
$$

where

$$
\begin{aligned}
& \boldsymbol{\Phi}_{2,1}^{T}=\left[\begin{array}{c}
-\mathbf{1}_{\left(N_{\mathcal{T}} \times N_{\mathcal{I}_{r}}\right)} \\
\mathbf{0}_{\left(N_{P} \times N_{\mathcal{I}_{r}}\right)}
\end{array}\right], \boldsymbol{\Phi}_{2,2}^{T}=\left[\begin{array}{c}
\mathbf{0}_{\left(N_{\mathcal{T}} \times N_{\mathcal{I}_{s}}\right)} \\
-\operatorname{Diag}^{\left(N_{\mathcal{I}_{s}}\right)}\left(\mathbf{1}_{N_{\mathcal{T}}}\right) \\
\mathbf{0}_{\left(N_{\mathcal{T}} \times N_{\mathcal{I}_{s}}\right)}
\end{array}\right] \\
& \boldsymbol{\Phi}_{2,3}^{T}=\left[\begin{array}{c}
\operatorname{Diag}(\beta)_{\left(N_{\mathcal{T}} \times N_{\mathcal{T}}\right)} \\
\mathbf{0}_{\left(\left[N_{\mathcal{T}} \times N_{\mathcal{I}_{s}}\right] \times N_{\mathcal{T}}\right)} \\
-\mathbf{I}_{N_{\mathcal{T}}}
\end{array}\right], \boldsymbol{\Phi}_{2,4}^{T}=\left[\begin{array}{c}
-\mathbf{1}_{N_{P}}^{(\mathrm{DR})} \\
\mathbf{0}_{N_{\mathcal{T}}}
\end{array}\right],
\end{aligned}
$$

and

$$
\boldsymbol{\Phi}_{3\left(\left[N_{P}+N_{A}\right] \times\left[N_{B_{c}}+N_{B_{a}}\right]\right)}^{T}=\left[\begin{array}{cc}
\mathbf{Q}_{t\left(N_{P} \times N_{B_{c}}\right)}^{T} & \mathbf{0}_{\left(N_{P} \times N_{B_{a}}\right)}^{T} \\
\mathbf{0}_{\left(N_{A} \times N_{B_{c}}\right)}^{T} & \mathbf{S}_{t\left(N_{A} \times N_{B_{a}}\right)}^{T}
\end{array}\right] .
$$

365 Here, the vector $\mathbf{1}_{N_{P}}^{(\mathrm{DR})}$ in $\boldsymbol{\Phi}_{2,4}^{T}$ represents a vector with ones and zeros at the positions corresponding to the DR period and non-DR period, respectively. $\operatorname{Diag}(a)$ represents a diagonal matrix with an element $a$. The matrices $\mathbf{Q}_{t}$ and $\mathbf{S}_{t}$ are the truncated matrices of $\mathbf{Q}$ and $\mathbf{S}$, corresponding to each binding constraints. $N_{e q}$ is a total number of equality constraints. 
The first two matrices correspond to the perturbed objective functions with respect to data:

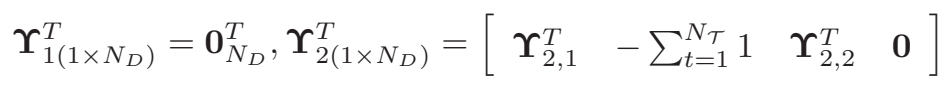

where

$$
\boldsymbol{\Upsilon}_{2,1\left(1 \times N_{\mathcal{T}}\right)}^{T}=\sum_{t=1}^{N_{\mathcal{T}}}\left(A_{t}^{* \mathrm{in}}-A^{\mathrm{comf}}\right)^{2}, \boldsymbol{\Upsilon}_{2,2\left(1 \times N_{\mathcal{T}}\right)}^{T}=-\sum_{t=1}^{N_{\mathcal{T}}} 2 \omega_{1}\left(A_{t}^{* \mathrm{in}}-A^{\mathrm{comf}}\right)
$$

The former is the row vector with zeros because the electricity cost function has no data associated with the sensitivity analysis.

The other three matrices are associated with the perturbed KKT condition equations with respect to data:

$$
\begin{aligned}
& \Upsilon_{3\left(\left[N_{P}+N_{A}\right] \times N_{D}\right)}^{T}= \\
& {\left[\begin{array}{ccccccc}
\mathbf{0}_{N_{P}} & \mathbf{0} & \mathbf{0} & \mathbf{0} & \mathbf{0} & \mathbf{0} & \mathbf{0} \\
\boldsymbol{\Upsilon}_{3,1}^{T} & \mathbf{0} & -2 \omega_{1} \mathbf{1}_{N_{\mathcal{T}}} & \mathbf{0} & \boldsymbol{\Upsilon}_{3,2}^{T} & \boldsymbol{\Upsilon}_{3,3}^{T} & \mathbf{0}
\end{array}\right]}
\end{aligned}
$$

where

$$
\begin{aligned}
& \Upsilon_{3,1\left(N_{\mathcal{T} \times 1)}\right.}^{T}=\left[2 \omega_{1}\left(A_{t}^{* \text { in }}-A^{\mathrm{comf}}\right)\right], \boldsymbol{\Upsilon}_{3,2}^{T}=-\left[\lambda_{2}^{*(3)}, \ldots, \lambda_{N_{\mathcal{T}}}^{*(3)}, 0\right]^{T}, \\
& \boldsymbol{\Upsilon}_{3,3}^{T}=\left[\lambda_{1}^{*(3)}, \ldots, \lambda_{N_{\mathcal{T}}}^{*(3)}\right]^{T},
\end{aligned}
$$

and

$$
\begin{aligned}
& \boldsymbol{\Upsilon}_{4\left(\left[N_{P}+N_{A}\right] \times N_{D}\right)}^{T}= \\
& {\left[\begin{array}{cccccccc}
0 & -1 & \mathbf{0} & \mathbf{0} & \mathbf{0} & \mathbf{0} & \mathbf{1} & \mathbf{0} \\
0 & -1 & \mathbf{0} & \mathbf{0} & \mathbf{0} & \mathbf{0} & \mathbf{0} & \mathbf{0} \\
0 & -1 & \mathbf{0} & \boldsymbol{\Upsilon}_{4,1}^{T} & \boldsymbol{\Upsilon}_{4,2}^{T} & -\alpha \mathbf{I}_{N_{\mathcal{T}}} & \mathbf{0} & \mathbf{0} \\
0 & 0 & -\mathbf{1} & \mathbf{0} & \mathbf{0} & \mathbf{0} & \mathbf{1} & \mathbf{0}
\end{array}\right]}
\end{aligned}
$$

where

$$
\mathbf{\Upsilon}_{4,1\left(N_{\mathcal{T} \times 1)}^{T}\right.}^{T}=\left[\left(A_{t-1}^{* \mathrm{in}}-\widehat{A}_{t}^{\text {out }}\right)\right], \mathbf{\Upsilon}_{4,2\left(N_{\mathcal{T} \times 1)}^{T}\right.}^{T}=\left[-P_{i, t}^{*}\right]
$$

and 


$$
\mathbf{\Upsilon}_{5\left(\left[N_{B_{c}}+N_{B_{a}}\right] \times N_{D}\right)}^{T}=\left[\begin{array}{ccc}
\mathbf{0} & -\mathbf{Q}_{t\left(N_{B_{c}} \times N_{P}\right)} & \mathbf{0} \\
\mathbf{0} & \mathbf{0} & -\mathbf{Q}_{t\left(N_{B_{c}} \times N_{P}\right)}
\end{array}\right]
$$

\section{References}

[1] J. Deign, C. M. Salazar, Da a management and analytics for utilities, Technical Report.

URL htt.p://www.smartgridupdate.com/dataforutilities/pdf/DataManagementWhitePaper.pdf

[2] A. Saha, S. Rahman, M. Pipattanasomporn, M. Kuzlu, On security of a home energy management system, 2014 IEEE PES Innovative Smart Grid Technologies Conference Europe (2014) 1-5.

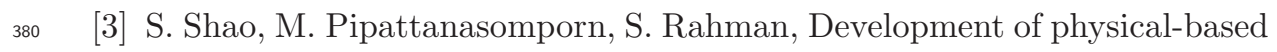
demand response-enabled residential load models, IEEE Transactions on Power Systems 28 (2) (2013) 607-614. doj:10.1109/TPWRS.2012.2208232.

[4] M. Pipattanasomporn, M. Kuzlu, S. Rahman, An algorithm for intelligent home energy management and demand response analysis, IEEE Transactions on Smart Grid 3 (4) (2012) 2166-2173. doj: $10.1109 /$ TSG.2012.2201182.

[5] M. A. A. Pedrasa, T. D. Spooner, I. F. MacGill, Coordinated scheduling of residential distributed enrgy resource to optimize smart home energy services, IEEE Transactions on Smart Grid 1 (2) (2012) 134-143. doi :10.1109/TSG.2010.2053053.

[6] O. Erdinc, N. G. Paterakis, T. D. P. Mendes, A. G. Bakirtzis, J. P. S. Catalão, Smart household operation considering bi-directional EV and ESS utilization by real-time pricing-based DR, IEEE Transactions on Smart Grid 6 (3) (2015) 1281-1291. doj:10.1109/TSG.2014.2352650. 

method for hoursehold load scheduling considering uncertainties of customer behavior, IEEE Transactions on Smart Grid 6 (4) (2015) 1806-1818. doi:10.1109/TSG.2015.2403411.

[8] M. Rastegar, M. F. Firuzabad, Outage management in residential demand response programs, IEEE Transactions on Smart Grid 6 (3) (2015) 14531462. doi:10.1109/TSG.2014.2338794

[9] S. Lee, B. Kwon, S. Lee, Joint energy management system of electric supply and demand in houses and buildings, IEEE Transactions on Power Systems 29 (6) (2014) 2804-2812. doi:10.1109/TPWRS.2014.2311827.

[10] D. T. Nguyen, L. B. Le, Joint optimization of electric vehicle and home energy scheduling considering user comfort preference, IEEE Transactions on Smart Grid 5 (1) (2014) 188-199. doi:10.1109/TSG.2013.2274521.

[11] K. M. Tsui, S. C. Chan, Demand response optimization for smart home scheduling under real-time pricing, IEEE Transactions on Smart Grid 3 (4) (2012) 1812-1821. doi:10.1109/TSG.2012.2218835.

[12] A. A. Moghaddam, H. Monsef, A. R. Kian, Optimal smart home energy management considering energy saving and a comfortable lifestyle, IEEE Transactions on Smart Grid 6 (1) (2015) 324-332. doi:10.1109/TSG.2014.2349352

[13] P. Chavali, P. Yang, A. Nehorai, A distributed algorithm of appliance scheduling for home energy management system, IEEE Transactions on Smart Grid 5 (1) (2014) 282-290. doi:10.1109/TSG.2013.2291003.

[14] N. Li, L. Chen, S. H. Low, Optimal demand response based on utility maximization in power networks, 2011 IEEE Power and Energy Society General Meeting (2011) 1-8.

[15] I. Ullah, N. Javaid, M. Imran, J. A. Khan, U. Qasim, M. Alnuem, M. Bashir, A survey of home energy management for residential customers, 
2015 IEEE 29th International Conference on Advanced Information Networking and Applications (2015) 666-673.

${ }_{425}[16]$ Z. Pan, Q. Guo, H. Sun, Impacts of optimization interval on home energy scheduling for thermostatically controlled appliances, CSEE Journal of Power and Energy Systems 1 (2) (2015) 90-100. doi:10.17775/CSEEJPES.2015.00024

[17] O. Elma, U. S. Selamogullari, A new home energy management algorithm with voltage control in a smart home environment, Energy 6 (2015) 720731. doi:10.1016/j.energy.2015.08.094.

[18] Y. Xia, S. G. Ghiocel, D. Dotta, D. Shawhan, A. Kindle, J. H. Chow, A simultaneous perturbation approach for solving economic dispatch problems with emission, storage, and network constraints, IEEE Transactions on Smart Grid 4 (4) (2013) 2356-2363. doi:10.1109/TSG.2014.2338794.

[19] F. Li, R. Bo, DCOPF-based LMP simulation: Algorithm, comparisonwith acopf, and sensitivity, IEEE Transactions on Power Systems 22 (4) (2007) 1475-1485. doi:10.1109/TPWRS.2007.907924.

[20] A. J. Conejo, E. Castillo, R. Minguez, F. Milano, Locational marginal price sensitivities, IEEE Transactions on Power Systems 20 (4) (2005) 2026-2033. doi:10.1109/TPWRS.2005.857918.

[21] E. Castillo, A. J. Conejo, C. Castillo, R. Mínguez, D. Ortigosa, Perturbation approach to sensibility analysis in mathematical programming, Journal of Optimization Theory and Applications 128 (1). doi:10.1007/s10957-005-7557-y

[22] N. Arghira, L. Hawarah, S. Ploix, M. Jacomino, Prediction of appliances energy use in smart homes, Energy 48 (2012) 128-134. doi:10.1016/j.energy.2012.04.010 


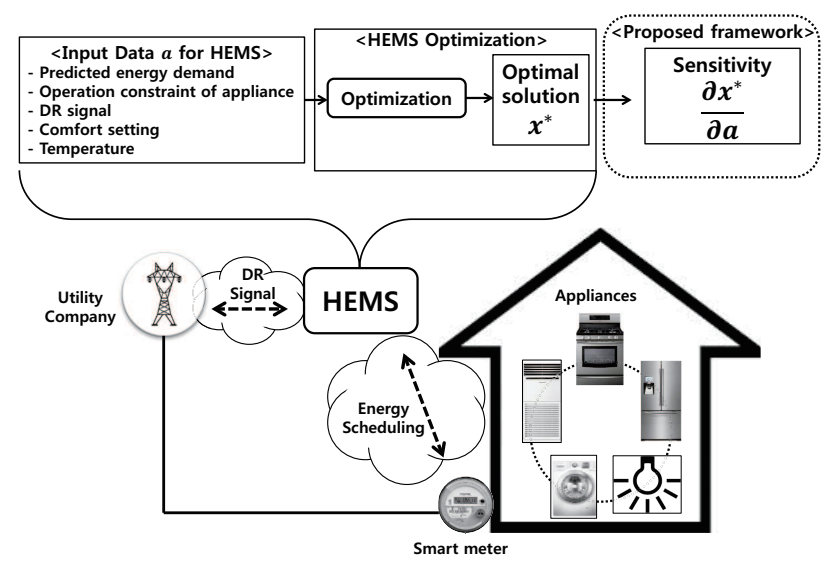

Figure 1: Conceptual system model for HEMS.

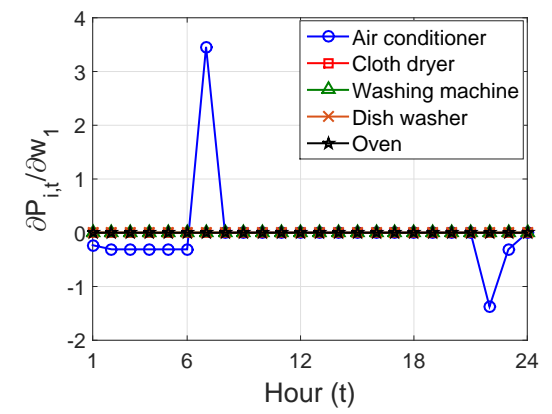

(a)

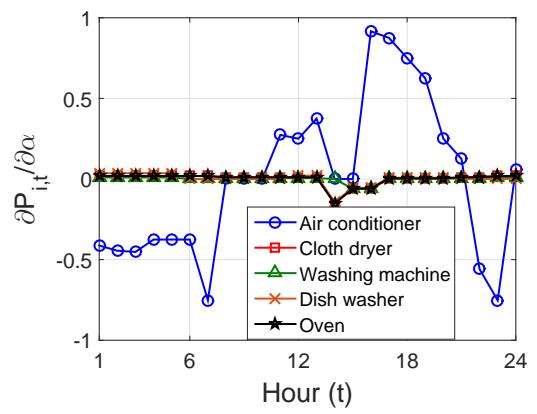

(b)

Figure 2: Sensitivities of $P_{i, t}$ with respect to the change in: (a) $\omega_{1}$ and (b) $\alpha$.

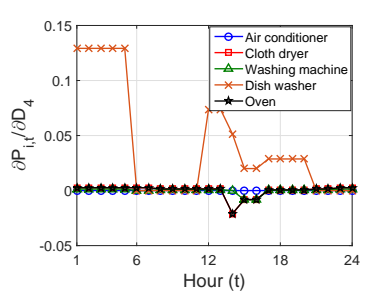

(a)

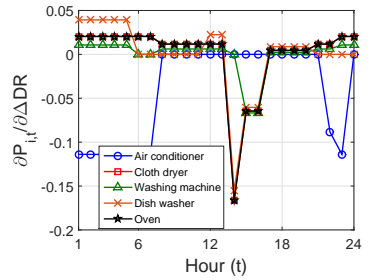

(b)

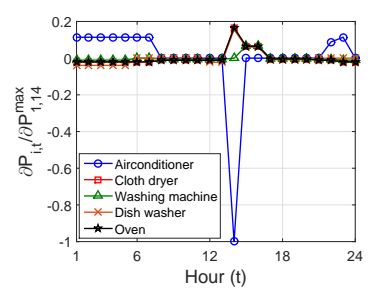

(c)

Figure 3: Sensitivities of $P_{i, t}$ with respect to the change in: (a) $\widehat{D}_{4}$, (b) $\Delta \mathrm{DR}$, and (c) $P_{1,14}^{\max }$. 
Table 1: Notation

\begin{tabular}{|c|c|}
\hline$i$ & Index for appliance $i$ \\
\hline$t$ & Index for time slot $t$ \\
\hline$P_{i, t}$ & Power consumption of appliance $i$ at time slot $t$ \\
\hline$P_{i, t}^{\max (\min )}$ & Maximum(Minimum) power consumption of appliance $i$ at time slot $t$ \\
\hline$A_{i, t}^{\mathrm{in}}$ & Indoor temperature by appliance $i$ at time slot $t$ \\
\hline$\widehat{A}_{t}^{\text {out }}$ & Predicted outdoor temperature at time slot $t$ \\
\hline$A^{\mathrm{comf}}$ & The most comfortable temperature set by the consumer \\
\hline$A^{\mathrm{in}, \max (\min )}$ & Maximum(Minimum) comfortable temperature \\
\hline$\alpha, \beta$ & Thermal characteristics for air conditioner \\
\hline$\widehat{D}_{i}$ & Predicted daily energy consumption of appliance $i$ \\
\hline$\widehat{D}_{t}$ & Predicted total energy consumption at time slot $t$ \\
\hline$\omega_{1}, \omega_{2}$ & Parameters for the consumer's dissatisfaction cost \\
\hline$\gamma_{i, t}$ & $\begin{array}{l}\text { Consumption state of appliance } i \text { at time slot } t \text {, " } 1 \text { " for consumption, " } 0 \text { " } \\
\text { otherwise at time } k\end{array}$ \\
\hline$\Delta \mathrm{DR}$ & Total requested demand reduction during DR period \\
\hline$C_{t}(\cdot)$ & Total cost function of energy consumption at time slot $t$ \\
\hline $\mathcal{I}$ & Set of appliances $\left(\mathcal{I}=\mathcal{I}_{r} \cup \mathcal{I}_{s}\right)$ \\
\hline $\mathcal{I}_{r}$ & Set of reducible appliances \\
\hline $\mathcal{I}_{s}$ & Set of shiftable appliances \\
\hline $\mathcal{T}$ & Set of time slots \\
\hline $\mathcal{T}_{\mathrm{DR}}$ & Set of DR time slots $\left(\mathcal{T}_{\mathrm{DR}} \subset \mathcal{T}\right)$ \\
\hline$N_{\mathcal{I}}$ & Total number of appliances \\
\hline$N_{\mathcal{I}_{r}}$ & Total number of reducible appliances \\
\hline$N_{\mathcal{I}_{s}}$ & Total number of shiftable appliances \\
\hline$N_{\mathcal{T}}$ & Total number of time slots for energy schedulin \\
\hline$N_{\mathcal{T}_{\mathrm{DR}}}$ & Total number of DR time slots \\
\hline$N_{P}$ & Total number of decision variables $P_{i, t}$ \\
\hline$N_{A}$ & Total number of decision variables $A_{t}^{\text {in }}$ \\
\hline$N_{D}$ & Total number of data \\
\hline $\mathbf{1}_{k}, \mathbf{0}_{k}$ & $k \times 1$ column vectors with all ones and all zeros, respectively \\
\hline $\mathbf{I}_{k}$ & $k \times k$ identity matrix \\
\hline
\end{tabular}


Table 2: Classification of data in HEMS

\begin{tabular}{ccc}
\hline Group & Description & Data \\
\hline (G1) & Predicted energy demand & $\widehat{\mathbf{D}}_{i}, \widehat{\mathbf{D}}_{t}$ \\
$(\mathrm{G} 2)$ & Operational constraint of appliance & $\alpha, \beta, \mathbf{P}^{\text {cap }}$ \\
$(\mathrm{G} 3)$ & DR signal & $\Delta \mathrm{DR}$ \\
$(\mathrm{G} 4)$ & Comfort setting & $\boldsymbol{\omega}, A^{\text {comf }}, \mathbf{A}^{\text {in,cap }}$ \\
$(\mathrm{G} 5)$ & Weather information & $\widehat{\mathbf{A}}^{\text {out }}$ \\
\hline
\end{tabular}

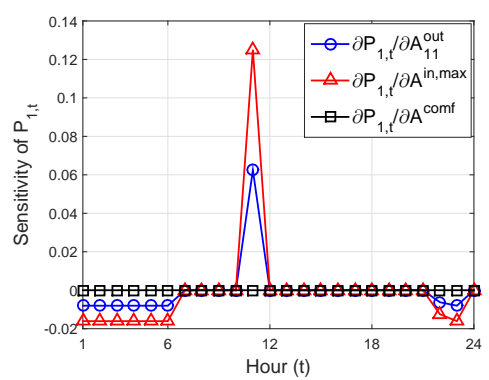

Figure 4: Sensitivities of $P_{1, t}$ with respect to the change in $\widehat{A}_{11}^{\text {out }}, A_{11}^{\text {in,max }}$ and $A^{\text {comf }}$.

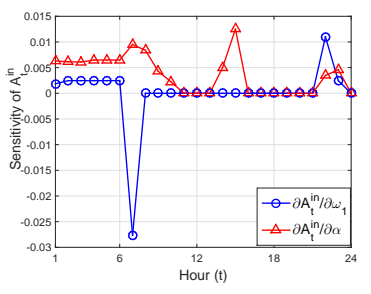

(a)

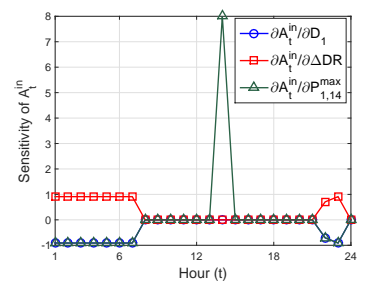

(b)

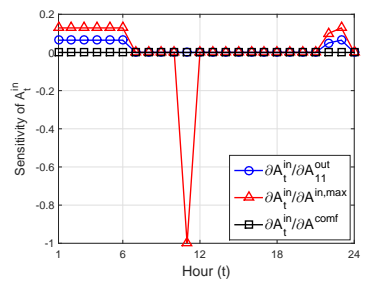

(c)

Figure 5: Sensitivities of $A_{t}^{\text {in }}$ with respect to the change in: (a) $\omega_{1}$ and $\alpha$, (b) $\widehat{D}_{1}, \Delta \mathrm{DR}$ and $P_{1,14}^{\max }$, and (c) $\widehat{A}_{11}^{\text {out }}, A^{\text {in, } \max }$ and $A^{\text {comf }}$. 


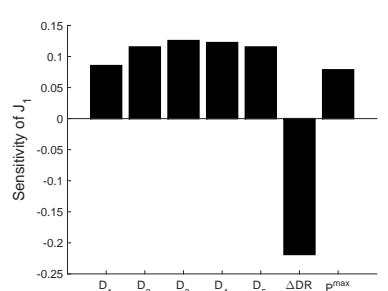

(a)

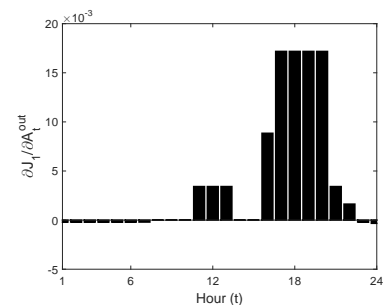

(c)

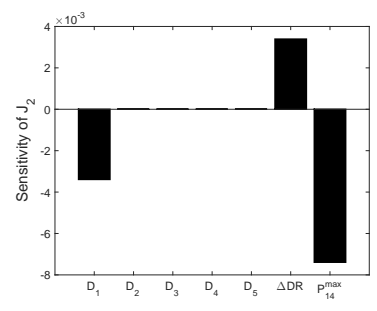

(b)

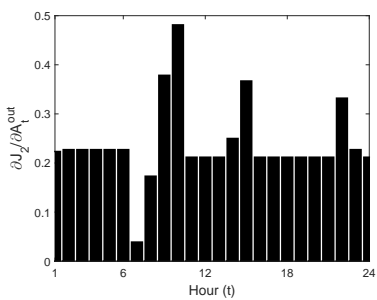

(d)

Figure 6: Sensitivities of $J_{1}$ and $J_{2}$ with respect to the change in: (a), (b) $\widehat{D}_{i}, \Delta \mathrm{DR}, P_{1,14}^{\max }$, and (c), (d) $\widehat{A}_{t}^{\text {out }}$. 